\title{
Grüne Gentechnik und Genome Editing
}

\section{Erfordernisse einer Neuausrichtung der Wissenschaftskommunikation}

\author{
Steffen Albrecht, Büro für Technikfolgen-Abschätzung beim Deutschen Bundestag (TAB), Neue Schönhauser Straße 10, \\ 10178 Berlin (albrecht@tab-beim-bundestag.de) \\ Julia Diekämper, Interdisziplinäre Arbeitsgruppe Gentechnologiebericht, Berlin-Brandenburgische \\ Akademie der Wissenschaften (BBAW) (diekaemper@bbaw.de) \\ Lilian Marx-Stölting, Interdisziplinäre Arbeitsgruppe Gentechnologiebericht, Berlin-Brandenburgische \\ Akademie der Wissenschaften (BBAW) (marx-stoelting@bbaw.de) \\ Arnold Sauter, Büro für Technikfolgen-Abschätzung beim Deutschen Bundestag (TAB) (sauter@tab-beim-bundestag.de)
}

Genome Editing gilt als Technologiesprung in der Gentechnologie. Die potenzielle Reichweite der Verfahren und die damit verbundenen Chancen und Risiken geben Impulse für eine neue Runde in der Debatte über Gentechnologien, die vor allem im Bereich der Grünen Gentechnik bislang durch Lagerdenken und einen Mangel an Zwischenpositionen gekennzeichnet ist. Um in Zukunft sachgerechter und unter Einbeziehung unterschiedlicher gesellschaftlicher Interessen über Optionen der weiteren Entwicklung der neuen Technologien diskutieren zu können, werden in diesem Beitrag Anforderungen an eine Neuausrichtung der Wissenschaftskommunikation umrissen.

\section{"Green" Genetic Engineering and Genome Editing}

Towards a Reorientation of Science Communication

Genome editing is viewed as a breakthrough in genetic engineering. The potential scope of the procedures and the associated risks and opportunities give impetus to a new round in the public debate on genetic engineering, a debate that is characterized by profound opposition and the absence of intermediate positions, particularly in the field of agriculture. This article sketches some elements of science communication that are deemed necessary to discuss options for the further development of new technologies in a more appropriate manner and by taking into account different societal interests.

KEYWORDS: genetic engineering, science communication, genome editing, public discourse

\section{Genome Editing: neue Runde in der Wissenschaftskommunikation zur Gentechnik}

Genome Editing gilt als Technologiesprung in der Gentechnologie. Die neuen Verfahren werden als Möglichkeit gehandelt, schneller und gezielter in das Erbgut von Pflanzen, Tieren und auch Menschen einzugreifen, als es mithilfe bisheriger gentechnischer Methoden möglich war. Dadurch sind mittlerweile Forschungsdesigns und Anwendungsmöglichkeiten in greifbare Nähe gerückt, die früher praktisch nicht realisierbar waren. In der Forschung ist die Zahl der Anwendungen insbesondere des CRISPR/Cas9-Systems enorm gestiegen. Die Auszeichnung als Breakthrough of the Year durch das Magazin Science im Jahr 2015 macht die potenzielle Reichweite dieser Entwicklung deutlich.

In Anbetracht der weitreichenden Möglichkeiten der neuen Methoden sowie der mit ihnen verbundenen potenziellen $\mathrm{Ri}$ siken riefen WissenschaftlerInnen unterschiedlicher Disziplinen von Anfang an zu einer gesellschaftlichen Auseinandersetzung über Möglichkeiten und Grenzen, aber auch über Förderung bzw. Regulierung des Genome Editing auf (z. B. Baltimore et al. 2015; Lanphier et al. 2015; Reich et al. 2015; Dorn 2016; NASEM 2015, 2017a; Bonas et al. 2017). Solche Aufrufe erfolgten in den letzten Jahren regelmäßig im Zusammenhang mit neuen Technologien, nachdem technologische Risiken und Krisenereignisse das Bewusstsein dafür gestärkt hatten, dass wissenschaftliche Forschung und Technologieentwicklung nicht nur Probleme lösen, sondern im gleichen Zuge auch gesellschaftsweite Risiken schaffen können (Jasanoff 2003). Zunehmend wurde argumentiert, dass Entscheidungen über Fragen der Förderung und Regulierung von Forschung und Technologie der Legitimation durch eine gesellschaftliche Debatte bedürfen (Europäische Kommission 2009; Felt et al. 2013). Allerdings bleibt
This is an article distributed under the terms of the Creative Commons Attribution License CCBY 4.0 (https://creativecommons.org/licenses/by/4.0/) https://doi.org/10.14512/tatup.26.3.64

Submitted: 04. 08.2017. Peer reviewed. Accepted: 13.10.2017 
bei entsprechenden Aufrufen häufig (so auch im Fall des Genome Editing) unklar, welche Ziele mit einer öffentlichen Debatte im Einzelnen verfolgt werden und in welcher Form und durch wen sie angeregt und umfassend geführt werden soll.

Klar ist hingegen, dass der Wissenschaftskommunikation, verstanden als Schnittstelle zwischen wissenschaftlichen Akteuren und Institutionen einerseits und sonstigen gesellschaftlichen Akteuren andererseits, eine besondere Rolle zugeschrieben wird. Sie soll gemäß der Definition von Burns et al. (2003) mithilfe geeigneter Fähigkeiten, Medien und Aktivitäten das Bewusstsein und Interesse für Wissenschaft stärken, Freude an und Verständnis für Wissenschaft erzeugen sowie zur Meinungsbildung in wissenschaftsbezogenen Fragen beitragen. Ihre zunehmende Bedeutung wird am entsprechenden Engagement wissenschaftlicher Institutionen und Förderorganisationen deutlich, aber auch am vermehrten Interesse an ihr als Reflexions- und Forschungsgegenstand (siehe z. B. TATuP-Schwerpunkte 3/2005 und 1/2016 sowie Kähler 2017; NASEM 2017b; Bonfadelli et al. 2017; Weitze und Heckl 2016).

Doch wie kann die Wissenschaftskommunikation dazu beitragen, zwischen unterschiedlichen Zielstellungen, Sichtweisen, Wertvorstellungen und Interessen in Bezug auf Wissenschaft und Technologie zu vermitteln und einen konstruktiven und ergebnisoffenen Diskurs über diese zu ermöglichen? Im vorliegenden Beitrag möchten wir dieser Frage, die sich insbesondere auch Einrichtungen der Begleitforschung und Technikfolgenabschätzung stellt, mit Blick auf die Debatte über die Gentechnologie nachgehen. Wie wurde bzw. wird sie bislang (in Bezug auf Grüne Gentechnik) und aktuell (in Bezug auf Genome Editing) geführt? Auf der Basis einer Auseinandersetzung mit den dabei gemachten Erfahrungen beschreiben wir einige zentrale Herausforderungen der Gestaltung der Wissenschaftskommunikation zum Genome Editing.

\section{Ein Blick zurück: Was „lief falsch“ bei der Kommunikation der Grünen Gentechnik?}

Das gesellschaftlich am meisten umkämpfte Anwendungsfeld der Gentechnologie ist fraglos die so genannte Grüne Gentechnik, also die Anwendung der Gentechnik in der Landwirtschaft. Die öffentliche Diskussion um sie ist durch ausgeprägtes Lagerdenken sowie einen Mangel an Zwischenpositionen geprägt. Ein sachlicher Austausch zwischen den Lagern in Form einer Diskussion über die Vor- und Nachteile unterschiedlicher Arten der Züchtung und über alternative Entwicklungspfade der Technologie findet praktisch nicht statt.

Doch welche Rolle hat die Wissenschaftskommunikation in der gesellschaftlichen Debatte bisher gespielt? Es lassen sich verschiedene Ansätze erkennen, die offenkundig nicht überzeugend waren, wie etwa der Versuch der Positionierung von WissenschaftlerInnen als objektive Autoritäten (ausführlicher z. B. Weitze et al. 2012). Tatsächlich wurden und werden viele Wis-
senschaftlerInnen von der Öffentlichkeit als der Politik bzw. Wirtschaft nahe stehend wahrgenommen und haben ja auch häufig ein eigenes Interesse an der weiteren Erforschung und Förderung der Gentechnologie.

Ein Negativbeispiel bieten immer wieder prominente WissenschaftlerInnen, die sich für die Grüne Gentechnik aussprechen, obwohl sie über keine spezifische Expertise verfügen, die ihnen eine besondere Kompetenz für dieses Thema verleihen würde. Zuletzt warben etwa im Sommer 2016 mehr als 100 NobelpreisträgerInnen dafür, die Entwicklung von nährstoffoptimiertem Reis (golden rice) zu forcieren (Nestler 2016). Das Problem des Mikronährstoffmangels wurde dabei stark vereinfacht,

\section{Ein sachlicher Austausch zwischen den Lagern findet bei der Grünen Gentechnik praktisch nicht statt.}

der goldene Reis als alternativlose Methode zu dessen Bekämpfung präsentiert, seine Nichtnutzung als künftiges ,Verbrechen gegen die Menschlichkeit" dargestellt (o. A. 2016). Eine solche Positionierung erscheint sowohl undifferenziert als auch in fragwürdiger Weise moralisierend (Karberg 2016). Das Vertrauen in die Expertise der Unterzeichnenden auf diese Weise auszunutzen, kann das Ansehen von Wissenschaft und Forschung insgesamt beschädigen.

Versprechungen zu den Potenzialen der Gentechnik, die von Befürwortenden nicht belegt werden konnten, erwiesen sich ebenfalls als Belastung für das Vertrauen in die Wissenschaft. ${ }^{1}$ Die tatsächlich entwickelten Produkte (überwiegend Pflanzensorten mit Herbizidtoleranz und Insektenresistenz) hatten für EndverbraucherInnen keinen Nutzen. Auch das erste Endverbraucherprodukt, die Flavr-Savr-Tomate (umgangssprachlich „Anti-Matsch-Tomate"), war nicht geeignet, die Relevanz der Grünen Gentechnik für die Bevölkerung sichtbar zu machen die Tomate wies zwar eine vergleichsweise lange Haltbarkeit auf, erhöhte aber den Transportaufwand (Martineau 2001) und endete hauptsächlich als Bestandteil von Tomatenmark, bevor sie nach kurzer Zeit wieder vom Markt genommen wurde. Als Ziel der Anwendung von Gentechnik wurde immer wieder pauschal die Lösung des Welternährungsproblems in Aussicht gestellt, ohne dass plausible Gesamtstrategien hierfür präsentiert wurden. In Diskussionsbeiträgen wurde zudem nicht genügend nach dem Stand der Forschung (bzw. Anwendung) differenziert - häufig ging es um frühe Stadien der Entwicklung, die noch weit von einer Anwendung entfernt waren bzw. dies noch immer sind, etwa bei dem Hinweis auf den Nutzen trockentoleranter Pflanzen.

1 Zu den Einstellungen von Verbrauchern gegenüber der Grünen Gentechnik sowie zur Konfliktdynamik siehe Hampel (2008) und Conrad (2008). 
Problematisch war in der Vergangenheit auch die Erwartung vieler WissenschaftlerInnen, es reiche aus, als unwissend wahrgenommene Laien mit Fakten zu informieren und allein dadurch eine größere Akzeptanz für ihre Forschung zu bewirken. Diese als „Defizitmodell“ bekannt gewordene Annahme prägte lange Zeit die Wissenschaftskommunikation weit über die Gentechnik hinaus. Obwohl sie eigentlich als überholt gilt, hält sie sich in weiten Teilen bis heute (Cortassa 2016; Bauer 2017). Dass die Haltung zur Gentechnik jedoch keine Frage des Wissens(defizits) ist und mehr Wissen nicht notwendigerweise zu mehr Akzeptanz führt, lässt sich nicht zuletzt daran ablesen, dass sich gerade unter Gebildeten oft vehemente Kritiker der Gentechnologie finden. Da in Bezug auf die Gentechnik grundlegende Interessen und Werte zur Debatte stehen, können WissenschaftlerInnen gegenüber anderen Diskussionsteilnehmenden in dieser Hinsicht keine spezielle Kompetenz für sich beanspruchen, und es sind neue Formate gefragt, den Austausch darüber zu strukturieren.

\section{Genome Editing - aktuelle Debatten}

Die neuen Möglichkeiten des Genome Editing werden vielfach als Revolution in der Gentechnik bezeichnet, womit auf die massive Ausweitung der Grundlagen- und der Anwendungsforschung sowie auf die möglichen gesellschaftlichen Folgen verwiesen wird. Doch sind damit auch neue Impulse für die festgefahrene Debatte über Gentechnologie verbunden? Zumindest lässt sich bereits in diesem frühen Stadium der Entwicklung beobachten, dass ganz unterschiedliche Akteure kommunikative Aktivitäten initiieren. Neben WissenschaftlerInnen suchen insbesondere die wissenschaftlichen Akademien gezielt den Kontakt zur Öffentlichkeit, so unter anderem die Berlin-Brandenburgische Akademie der Wissenschaften oder die Leopoldina mit Veranstaltungen zur Gentechnologie und zur Veränderbarkeit des Genoms. Auch das BMBF und andere Behörden sowie zivilgesellschaftliche Organisationen wie der Deutsche Evangelische Kirchentag führen Veranstaltungen durch oder fördern Projekte.

Das Thema Genome Editing wird auch in den Massenmedien aufgegriffen. Die Presseberichterstattung ${ }^{2}$ zeigt, dass im Zeitraum 2015 bis 2016 die Zahl der Artikel in auflagenstarken, überregionalen Zeitschriften und Zeitungen über alle Anwendungsfelder hinweg anstieg. Medizinische bzw. therapeutische Anwendungen bilden dabei einen zentralen Fokus. Charakteristisch für die Auseinandersetzung mit dem Genome Editing ist, dass die Felder der medizinischen und landwirtschaftlichen Anwendung (Rote und Grüne Gentechnik) beim Genome Editing zum Teil gemeinsam verhandelt werden, wie es in früheren

2 Die Ergebnisse der Medienanalyse (FAZ, ZEIT, SZ, Spiegel) wurden von Julia Diekämper und Lilian Marx-Stölting am 22.11.2016 auf einem Workshop von TAB und IAG Gentechnologiebeicht präsentiert, deren Schlussfolgerungen werden im „Vierten Gentechnologiebericht“ (erscheint 2018) veröffentlicht.
Entwicklungsstadien der ,konventionellen“ Gentechnik bereits der Fall war (Deutscher Bundestag 1987).

Neben den Potenzialen werden auch Risiken thematisiert, beispielsweise die Häufigkeit von Off-Target-Effekten, also nicht intendierten Veränderungen des Genoms an anderen Orten als der Zielsequenz, oder die Gefahren, die sich aus der Anwendung durch Do-it-yourself-Biologen außerhalb institutioneller Labors ergeben, insbesondere die Möglichkeit unbeabsichtigter Freisetzungen (Bio-Error) oder missbräuchlicher bzw. illegaler Verwendung biologischen Wissens (Bio-Terror) (Sauter et al. 2015). Im Unterschied zur (medialen) Diskussion über Grüne Gentechnik wird die CRISPR/Cas9-Technik außerdem weniger mit großen Unternehmen in Verbindung gebracht (eine Ausnahme ist die Fusion der Konzerne Bayer und Monsanto), sondern als Verfahren charakterisiert, das einer großen Zahl ganz unterschiedlicher Akteure zur Verfügung steht. Eine solche Darstellung greift das Narrativ der Einfachheit und Preisgünstigkeit gegenüber früheren Methoden der Gentechnik auf, das den Auftakt der meisten Artikel zum Genome Editing bildet.

Die mediale Berichterstattung wie auch die verschiedenen Initiativen zum Genome Editing erreichen bislang jedoch offenbar nicht die breite Bevölkerung. Einer Umfrage vom Juni 2016 (YouGov 2016) zufolge hatten zwar 29\% der deutschen Erwachsenen schon einmal die Begriffe CRISPR/Cas9 oder Genome Editing gehört. Doch nur 7\% gaben an zu wissen, was sich dahinter verbirgt. Auch im BfR-Verbraucher-Monitor Anfang 2017 gaben nur $14 \%$ der Befragten an, bereits vom Thema Genome Editing gehört zu haben (BfR 2017a). Sowohl in der YouGov-Befragung als auch in einer vom BfR durchgeführten qualitativen (Fokusgruppen-)Studie (BfR 2017b) zeigte sich, dass Genome Editing von vielen als eine Form der (bekannten) Gentechnik betrachtet wird und dem Thema mit den inzwischen etablierten Vorbehalten begegnet wird.

Dennoch wäre es falsch, die Debatte über Genome Editing mit der über die Gentechnologie komplett gleichzusetzen. Zwar sind Parallelen und Bezüge unverkennbar, etwa wenn in Bezug auf den Einsatz des Genome Editing in der Pflanzenzüchtung bekannte Pro- und Contra-Positionen reproduziert werden oder mit Blick auf die somatische Gentherapie auf die Probleme der früheren Methoden verwiesen wird. Aber auch neue Facetten und Impulse lassen sich erkennen. Beispiele dafür sind frühere Kritiker der Gentechnologie, die für eine Neubewertung des Genome Editing in der Pflanzenzucht plädieren (Maurin und Niggli 2016), sowie Regulierungsfragen, die entstehen, weil die neuen Anwendungsbereiche durch die bestehenden Gesetze nicht mehr ausreichend erfasst werden (Zinkant 2016). Das stärkere Bemühen vieler WissenschaftlerInnen und Wissenschaftsorganisationen um eine breite und ergebnisoffene gesellschaftliche Diskussion der Chancen und Risiken im Vorfeld der weiteren Erforschung schließlich legt die Annahme nahe, dass die Wichtigkeit der demokratischen Legitimierung von Forschung zunehmend anerkannt wird.

Inwiefern dabei neue Formen der Wissenschaftskommunikation entwickelt und genutzt werden, bleibt abzuwarten. Die ge- 
ringe Resonanz der aufgeworfenen Fragen in der Öffentlichkeit verweist auf das Problem, dass bislang nur ausgewählte Zielgruppen durch die genutzten Formate angesprochen wurden. Es bleibt zu evaluieren, welche gesellschaftlichen Gruppen sich aus welchen Gründen offenbar nicht angesprochen fühlen.

\section{Anforderungen an eine (Neuausrichtung der) Wissenschaftskommunikation zum Genome Editing}

Es ist angesichts der in Aussicht gestellten Potenziale des Genome Editing absehbar, dass ein großes öffentliches Interesse die (Weiter-)Entwicklung der Verfahren begleiten wird (Sauter et al. 2015). Aus unserer Sicht ist wünschenswert, dass eine möglichst große Zahl gesellschaftlicher Interessensgruppen z. B. mittels Konsultation oder Bürgerbeteiligung an der Debatte teilhat, damit die unterschiedlichen möglichen Entwicklungspfade der Technologie erkundet und ihre Chancen und Risiken jeweils abgeschätzt werden können. Nur so wird die Forschungsund Entwicklungsarbeit an gesellschaftliche Problemlagen und Wertvorstellungen gekoppelt und die Debatte bleibt offen für unterschiedliche Anknüpfungsmöglichkeiten. Die folgenden fünf Punkte fassen einige zentrale An- bzw. Aufforderungen an die Gestaltung der Wissenschaftskommunikation zum Genome Editing zusammen.

\section{Die Erwartungen an die Rolle der Wissenschafts- kommunikation sollten neu justiert werden.}

Das Genome Editing eröffnet prinzipiell die Möglichkeit für eine Neuorientierung der Debatte über die Gentechnologie, allerdings müssen dabei die Veränderungen der Rolle von Wissenschaft in der Gesellschaft berücksichtigt werden. Für Wissenschaftlichkeit als Wert kann in gesellschaftlichen Debatten heute kein bevorzugter Platz gegenüber anderen Werten und Normen mehr beansprucht werden. Dies gilt für das Genome Editing mit seinen vielfältigen Anwendungsfeldern, insbesondere auch beim Menschen, mindestens so sehr wie für die Gentechnik bisher. Vor diesem Hintergrund ergibt sich unserer Einschätzung nach für die Wissenschaftskommunikation eine ähnliche Aufgabe wie für TA als Politikberatung, nämlich nicht vorrangig Öffentlichkeitsarbeit zu betreiben, sondern zwischen den unterschiedlichen Wertvorstellungen und Perspektiven zu vermitteln.

\section{Wissenschaftskommunikation sollte vom wissen- schaftlichen Belohnungssystem gewürdigt werden.}

Ein stärkerer Austausch von WissenschaftlerInnen mit und eine größere Offenheit gegenüber JournalistInnen wären hilfreich, um die Vielfalt wissenschaftlicher Perspektiven und Argumente stärker öffentlich bewusst zu machen und den Anschein einer einseitigen Positionierung wie in der Debatte um die Grüne Gentechnik zu vermeiden. Viele WissenschaftlerInnen nehmen ihre Rolle als Kommunikatoren aber noch immer kaum wahr, was auch an den strukturellen Rahmenbedingungen für die Forschung liegt, die diesen Einsatz nicht honorieren. Daher sollte ein kommunikatives Engagement von WissenschaftlerInnen stärker als karriererelevant gewürdigt werden - in Anerkennung des Umstandes, dass die dafür aufgebrachte Zeit zwangsläufig für andere Tätigkeiten fehlt. Auch die Forschungsförderorganisationen könnten in ihren Ausschreibungen Aktivitäten im Bereich Wissenschaftskommunikation honorieren oder sogar einfordern.

\section{Wissenschaftskommunikation sollte auch als anspruchsvoller Wissenschaftsjournalismus verstanden und betrieben werden.}

Die Kommunikation durch WissenschaftlerInnen oder wissenschaftliche Institutionen ist nur eine Facette der Wissenschaftskommunikation. Für einen umfassend informierten öffentlichen Diskurs über Forschung und Technologie ist explizit auch ein kritischer Blick auf Praktiken, Interessen und die Finanzierung von Wissenschaft und Forschung nötig, u. a. um unseriöse Forschung, unethische Praktiken oder auch undifferenzierte Aussagen und überzogene Ansprüche zu identifizieren und zu thematisieren. Diese wichtige Aufgabe kann am ehesten von einem anspruchsvollen Wissenschaftsjournalismus übernommen werden. Ein interessantes Format in diesem Zusammenhang sind Journalists in Residence, zu denen erste Erfahrungsberichte vorliegen (Ronzheimer 2016). Es darf allerdings nicht das Ziel sein, publizistische und wissenschaftliche Qualitätskriterien zugunsten einer größeren Reichweite beim Publikum preiszugeben, auch wenn der Anspruch sein sollte, die gesamte Gesellschaft zu erreichen.

\section{Neue Kommunikationsformate bedürfen weiterer Erprobung und Anwendung.}

Das verstärkte Interesse der letzten Jahre an Wissenschaftskommunikation hat bereits insofern Früchte getragen, als mittlerweile viele verschiedene Kommunikationsformate zur Verfügung stehen, nicht zuletzt aufgrund der medialen Ausdifferenzierung. Nachdem Printmedien nicht mehr in der Lage sind, die Gesamtbevölkerung anzusprechen, ermöglichen das Internet und insbesondere die sozialen Medien prinzipiell eine hochspezifische, multimediale und zielgruppengenaue Kommunikation sowie stärker partizipativ ausgerichtete Formate.

Für die Synthetische Biologie als Weiterentwicklung der Gentechnik wurde im Rahmen der explorativen Umsetzung des Konzepts von Responsible Research and Innovation (Schomberg 2012) in die Praxis eine ganze Reihe innovativer Formen des Dialogs und der Reflexion aktueller Forschung entwickelt und erprobt, beispielsweise Theater- und Filmfestivals (Albrecht et al. 2015). Allerdings zeigt sich bei all diesen Aktivitäten das Problem, dass die erreichbaren und zu einer Teilnahme motivierbaren gesellschaftlichen Gruppen sehr klein und meist ohnehin am jeweiligen Thema interessiert sind. Inwiefern sich mit neuen Formaten tatsächlich eine Ausweitung der Debatte in die Breite der Gesellschaft hinein erreichen lässt, ist eine offene Frage. 


\section{Aus Fehlern der Vergangenheit sollte stärker gelernt werden.}

Die Kontroverse um die Grüne Gentechnologie macht nicht zuletzt deutlich, wie vielschichtig die an sie gebundenen Konflikte sind und welche unterschiedlichen Dimensionen - seien sie strukturell, seien sie normativ, seien sie ökonomisch oder rechtlich - sie berührt. Wissenschaftskommunikation kann einen Beitrag zur konstruktiven Bearbeitung dieser Konflikte leisten, wenn sie sich dieser Komplexität annimmt und sich nicht als Popularisierungsinstanz abstrakter wissenschaftlicher Inhalte versteht. Dabei sollten frühere Debatten und Probleme stärker reflektiert und aus der Vergangenheit gelernt werden. Es hat sich bewährt, konkrete Problemlösungsansätze (mit ihrem jeweiligen Anwendungskontext) zu diskutieren, anstatt wenig definierte allgemeine Verbesserungspotenziale einer Technologie (z. B. widerstandsfähiger, ertragreicher, umweltfreundlicher, ressourcenschonender) zu betonen. Außerdem sollte stärker reflektiert werden, was mit Wissenschaftskommunikation erreicht werden kann und wo ihre Grenzen liegen.

\section{Ausblick}

Die Beobachtung und Reflexion der Debatten über Forschung und Technologieentwicklung gehören schon seit einiger Zeit zu den Aufgaben der Begleitforschung und Technikfolgenabschätzung. Sie werden umso wichtiger, wenn die unterschiedlichen Akteure ihre Aktivitäten im Bereich der Wissenschaftskommunikation verstärken und mit verschiedenen Formaten experimentieren. Gleichzeitig sind Einrichtungen wie die Interdisziplinäre Arbeitsgruppe Gentechnologiebericht (IAG) der Berlin-Brandenburgischen Akademie der Wissenschaften und das Büro für Technikfolgen-Abschätzung beim Deutschen Bundestag (TAB) zunehmend selbst gefordert, Technikfolgenabschätzung enger mit Wissenschaftskommunikation zu verbinden. Denn bei beiden Unternehmungen geht es darum, mittels einer intensiven $\mathrm{Zu}$ sammenarbeit unterschiedlicher Disziplinen und Kompetenzen wissenschaftlich-technische Entwicklungen in ihrer Komplexität und Bewertungssensibilität verständlich zu machen - und zwar nicht mit dem Ziel der Akzeptanzbeschaffung oder um lediglich Aufmerksamkeit zu erreichen, sondern um eine überparteiliche Informationsbasis für den Austausch zwischen unterschiedlichen Interessenträgern zu schaffen.

Eine engere Verknüpfung von Wissenschaftskommunikation und Technikfolgenabschätzung liegt daher nahe, wirft aber, wie in diesem Beitrag dargestellt, viele Fragen auf. Eine Auseinandersetzung mit der Thematik wird auch in die Bilanzierung des Umgangs mit Gentechnologien einfließen, die die IAG zum Ende ihrer siebzehnjährigen Laufzeit Ende 2018 erstellen wird. In Anbetracht der Vorläufigkeit der vorgebrachten Überlegungen möchten die Autorinnen und Autoren aber insbesondere auch die Leserschaft der TATuP auffordern, die hier begonnene Reflexion aufzugreifen und mit Reaktionen und eigenen Diskussionsbeiträgen fortzusetzen.

\section{Danksagungen}

Für wichtige Anregungen zu diesem Beitrag danken wir den Teilnehmenden des Workshops „Wie gelingt Wissenschaftskommunikation? Das Beispiel Genome Editing", den die IAG und das TAB im Herbst 2016 veranstalteten. Der Beitrag versteht sich nicht als Zusammenfassung der Ergebnisse des Workshops, sondern stellt zentrale Erkenntnisse aus der Perspektive der Autorinnen und Autoren dar mit dem Ziel, die Diskussion in den weiteren Kreis der TA-Community hineinzutragen und fortzusetzen. Außerdem danken wir den GutachterInnen der TATuP für wertvolle Anregungen.

\section{Literatur}

Albrecht, Steffen; Coenen, Christopher; König, Harald (2015): Enriching the Methodological Scope of Technology Assessment. Initial Insights from SYNENERGENE, the Mobilisation and Mutual Learning Action Plan on Synthetic Biology. In: Constanze Scherz, Tomáš Michalek, Leonhard Hennen, Lenka Hebáková, Julia Hahn und Stefanie B. Seitz (Hg.): The Next Horizon of Technology Assessment. Proceedings from the PACITA 2015 Conference in Berlin. Prague: Technology Centre ASCR, S. 151-156.

Baltimore, David et al. (2015): A Prudent Path Forward for Genomic Engineering and Germline Gene Modification. In: Science 348 (6230), S.36-38. DOI: 10.1126/ science.aab1028.

Bauer, Martin W. (2017): Kritische Beobachtungen zur Geschichte der Wissenschaftskommunikation. In: Heinz Bonfadelli, Birte Fähnrich, Corinna Lüthje, Jutta Milde, Markus Rhomberg und Mike S. Schäfer (Hg.): Forschungsfeld Wissenschaftskommunikation. Wiesbaden: Springer VS, S. 17-40. DOI: 10.1007/978-3-658-12898-2_2.

BfR - Bundesinstitut für Risikobewertung (2017 a): BfR-Verbrauchermonitor 2/2017. Berlin: Bundesinstitut für Risikobewertung

BfR (2017 b): Neue Technologien zur Modifikation des Genoms. Möglichkeiten, Grenzen und gesellschaftliche Herausforderungen. BfR-Symposium Neue Technologien zur Modifikation des Genoms - Möglichkeiten, Grenzen und gesellschaftliche Herausforderungen, 6. Dezember 2016, Berlin. Berlin: Bundesinstitut für Risikoforschung.

Bonas, Ulla et al. (2017): Ethische und rechtliche Beurteilung des genome editing in der Forschung an humanen Zellen. Halle: Leopoldina - Nationale Akademie der Wissenschaften.

Bonfadelli, Heinz; Fähnrich, Birte; Lüthje, Corinna; Milde, Jutta; Rhomberg, Markus; Schäfer, Mike S. (Hg.) (2017): Forschungsfeld Wissenschaftskommunikation. Wiesbaden: Springer VS. DOI: 10.1007/978-3-658-12898-2.

Burns, Terry W.; O'Connor, D. John; Stocklmayer, Susan M. (2003). Science Communication: A Contemporary Definition. Public Understanding of Science 12 (2), S. 183-202.

Conrad, Jobst (2008): Diskursdeterminanten und -wirkungen. Bedingungen und Grenzen von Wissenschaftskommunikation in der Grünen Gentechnik. In: Roger J. Busch und Gernot Prütz (Hg.): Biotechnologie in gesellschaftlicher Deutung. München: Herbert Utz, S. 29-58.

Cortassa, Carina (2016): In Science Communication, Why Does the Idea of a Public Deficit Always Return? The Eternal Recurrence of the Public Deficit. In: Public Understanding of Science 25 (4), S. 447-459. DOI: 10.1177/0963662516629745. Deutscher Bundestag (1987): Bericht der Enquete-Kommission „Chancen und Risiken der Gentechnologie“, Drucksache 10/6775. Berlin: Deutscher Bundestag.

Dorn, Thea (2016): Wo bleibt der Aufschrei? In: Die Zeit Nr. 27 vom 23. 06.2016, S. 39 
Europäische Kommission (Hg.) (2009): Challenging Futures of Science in Society: Emerging Trends and Cutting-Edge Issues. Brüssel: Europäische Kommission.

Felt, Ulrike et al. (2013): Science in Society: Caring for our Futures in Turbulent Times. European Science Foundation Science Policy Briefing Nr. 50, June 2013. Straßburg: European Science Foundation.

Hampel, Jürgen (2008): Der Konflikt um die Grüne Gentechnik. Diskursverfahren und öffentliche Meinung. In: Roger J. Busch und Gernot Prütz (Hg.): Biotechnologie in gesellschaftlicher Deutung. München: Herbert Utz, S. 59-90.

Jasanoff, Sheila (2003): Technologies of Humility: Citizen Participation in Governing Science. In: Minerva 41 (3), S. 223-244.

Kähler, Esther (2017): Der heiße Sommer der Wissenschaftskommunikation 2014 - ein Rückblick. Online verfügbar unter http://www.wissenschaftskommunikation.de/der-heisse-sommer-derwissenschaftskommunikation-2014-ein-rueckblick-5633, zuletzt aktualisiert am 26.06.17, zuletzt geprüft am 10.10.2017.

Karberg, Sascha (2016): Diffamierende Rhetorik hilft niemandem weiter. Kommentar. In: transkript 22 (11), S. 70.

Lanphier, Edward; Urnov, Fyodor; Haecker, Sarah Ehlen; Werner, Michael; Smolenski, Joanna (2015): Don't Edit the Human Germ Line. In: Nature 519, S. 410-411. DOI: 10.1038/519410a.

Martineau, Belinda (2001): Food Fight. The Short, Unhappy Life of the Flavr Savr Tomato. In: The Sciences 41 (2), S. 24-29.

Maurin, Jost; Niggli, Urs (2016): Ökoforscher über neue Gentech-Methode: „CRISPR hat großes Potenzial“. In: Die Tageszeitung vom 06. 04.2016, S. 3.

NASEM - National Academies of Sciences, Engineering, and Medicine (2015): International Summit on Human Gene Editing: A Global Discussion. Washington, DC: The National Academies Press. DOI: 10.17226/21913.

NASEM (2017 a): Human Genome Editing: Science, Ethics, and Governance. Washington, DC: The National Academies Press. DOI: 10.17226/24623.

NASEM (2017 b): Communicating Science Effectively: A Research Agenda. Washington, DC: The National Academies Press. DOI: 10.17226/23674.

Nestler, Ralf (2016): Nobelpreisträger fordern Greenpeace zum Umdenken auf. In: Der Tagesspiegel Online vom 05. 07.2016, www.tagesspiegel.de/wissen/ gruene-gentechnik-nobelpreistraeger-fordern-greenpeace-zum-umdenkenauf/13809758.html, zuletzt geprüft am 04.10.2017.

o. A. (2016): Laureates Letter Supporting Precision Agriculture (GMOS). Online verfügbar unter http://supportprecisionagriculture.org/nobel-laureategmo-letter_rjr.html, zuletzt aktualisiert am 29.06.2016, zuletzt geprüft am 10.10.2017.

Reich, Jens et al. (2015): Genomchirurgie beim Menschen. Zur verantwortlichen Bewertung einer neuen Technologie. Berlin: Berlin-Brandenburgische Akademie der Wissenschaften.

Ronzheimer, Manfred (2016): Die Erneuerung des Innovationsjournalismus in Deutschland. Unveröffentlichter Abschlussbericht, Journalist in Residence. München: Max-Planck-Institut für Innovation und Wettbewerb (erhältlich auf Anfrage beim Autor).

Sauter, Arnold et al. (2015): Synthetische Biologie. Die nächste Stufe der Biotechnologie. TAB-Arbeitsbericht Nr.164. Berlin: Büro für TechnikfolgenAbschätzung beim Deutschen Bundestag.

Schomberg, René von (2012): Prospects for Technology Assessment in a Framework of Responsible Research and Innovation. In: Marc Dusseldorp und Richard Beecroft (Hg.): Technikfolgen abschätzen lehren. Bildungspotenziale transdisziplinärer Methoden. Wiesbaden: Springer VS, S. 39-61.
Weitze, Marc-Denis et al. (Hg.) (2012): Biotechnologie-Kommunikation. Kontroversen, Analysen, Aktivitäten. München: acatech - Deutsche Akademie der Technikwissenschaften.

Weitze, Marc-Denis; Heckl, Wolfgang M. (2016): Wissenschaftskommunikation. Schlüsselideen, Akteure, Fallbeispiele. Heidelberg: Springer.

YouGov (2016): „Genome Editing“ bei Lebensmitteln. Forschungssensation oder Konsumentenkatastrophe? YouGov Reports, August 2016.

Zinkant, Kathrin (2016): Dafür oder dagegen? In: Süddeutsche Zeitung vom 10.10.2016, S. 16

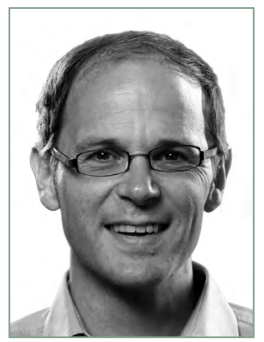

\section{DR. STEFFEN ALBRECHT}

ist Soziologe und seit 2014 beim Büro für Technikfolgen-Abschätzung beim Deutschen Bundestag tätig. Nach Studium und Promotion forschte er in Hamburg, Berlin, Dresden und Karlsruhe in verschiedenen Projekten zu den gesellschaftlichen Auswirkungen der Digitalisierung und zu politischer Kommunikation und Partizipation.

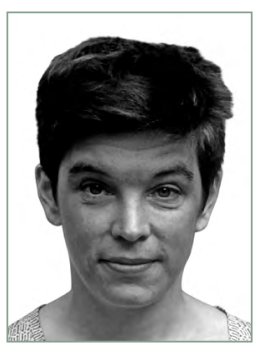

\section{DR. JULIA DIEKÄMPER}

ist Kulturwissenschaftlerin. Sie ist wissenschaftliche Mitarbeiterin der Berlin-Brandenburgischen Akademie der Wissenschaften und zudem Mitarbeiterin im Forschungsverbund "GenomELECTION". Julia Diekämper arbeitet als freie Autorin für Deutschlandfunk Kultur. Als Lehrbeauftragte war sie an unterschiedlichen Universitäten tätig.

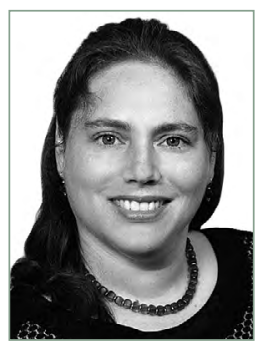

\section{DR. LILIAN MARX-STÖLTING}

ist Biologin und Bioethikerin und seit 2010 wissenschaftliche Mitarbeiterin der Interdisziplinären Arbeitsgruppe Gentechnologiebericht der BerlinBrandenburgischen Akademie der Wissenschaften. Außerdem lehrt sie Biologie und Bioethik in unterschiedlichen Kontexten und Formaten.

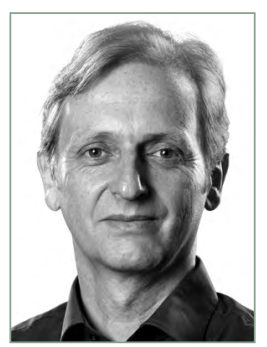

\section{DR. ARNOLD SAUTER}

ist seit 2012 stellvertretender Leiter des Büros für Technikfolgen-Abschätzung beim Deutschen Bundestag. Als Biologe befasst er sich seit seiner Promotion 1994 mit den Auswirkungen von Biound Medizintechnologien in ihren gesellschaftlichen und politischen Kontexten. Sein besonderes Interesse gilt Aushandlungsprozessen zwischen Wissenschaft, Politik, Wirtschaft, NGOS und weiteren Akteuren. 